\title{
Tests and Calibration of NIF Neutron Time of Flight Detectors
}

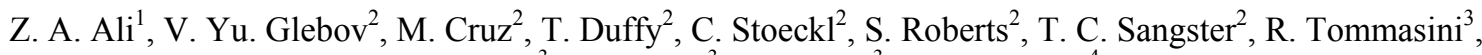

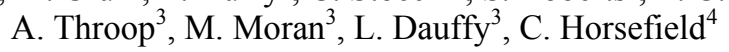 \\ ${ }^{I}$ National Security Technologies, LLC, Livermore CA \\ ${ }^{2}$ Laboratory for Laser Energetics, University of Rochester, Rochester NY \\ ${ }^{3}$ Lawrence Livermore National Laboratory, Livermore CA \\ ${ }^{4} \mathrm{AWE}$, United Kingdom
}

\begin{abstract}
The National Ignition Facility (NIF) Neutron Time of Flight (NTOF) diagnostic will measure neutron yield and ion temperature in all NIF campaigns in DD, DT, and THD* implosions. The NIF NTOF diagnostic is designed to measure neutron yield from $10^{9}$ to $2 \times 10^{19}$. The NTOF consists of several detectors of varying sensitivity located on the NIF at about $5 \mathrm{~m}$ and $20 \mathrm{~m}$ from the target. Production, testing, and calibration of the NIF NTOF detectors have begun at the Laboratory for Laser Energetics (LLE). Operational tests of the NTOF detectors were performed on several facilities including the OMEGA laser at LLE and the Titan laser at Lawrence Livermore National Laboratory (LLNL). Neutron calibrations were carried out on the OMEGA laser. Results of the NTOF detectors tests and calibration will be presented.

( $(\mathrm{D}=$ deuterium, $\mathrm{T}=$ tritium, $\mathrm{H}=$ hydrogen $)$

\section{Introduction}

Lawrence Livermore National Laboratory's (LLNL) National Ignition Facility (NIF) is a $1.8 \mathrm{MJ}$, 192-laser beam system designed to achieve nuclear fusion ignition $[1,2]$. The NIF NTOF, which will be an integral part of all NIF campaigns, will measure neutron yield and ion temperature of implosions in all NIF campaigns over the course of DD, DT, THD, and ignition experiments. It is designed to measure neutron yield from $10^{9}$ to $2 \times 10^{19}$. To provide the necessary dynamic range to measure this yield, the NTOF will consist of several detectors of varying sensitivity located on the NIF about $5 \mathrm{~m}$ and $20 \mathrm{~m}$ from the Target Chamber Center (TCC). Production, testing, and calibration of the NIF NTOF detectors have begun at the Laboratory for Laser Energetics (LLE) at the University of Rochester, with the support of the NSTec staff. Operational tests of the NTOF detectors were performed on the OMEGA laser at LLE, a multi-terawatt (MTW) laser at LLE, and on the Titan laser at LLNL. Calibrations of these detectors were performed on the OMEGA laser with the same electronics that will be used on the NIF. This paper describes results of initial tests and calibration of the NIF NTOF detectors.
\end{abstract}

\section{Detectors Design and Operational Testing}

The NIF NTOF detectors are of two basic types: (1) scintillator and photomultiplier tube (PMT) or vacuum photodiode (PD) assemblies, and (2) chemical vapor deposition (CVD) fabricated diamond detectors [3]. The current design, location, and neutron yield range of the NIF NTOF detectors are shown in Table 1. 
Table 1. NIF NTOF Detectors

\begin{tabular}{|c|c|c|c|c|c|}
\hline \# & Detector & Distance & PMT, gain & Campaign & Yield range \\
\hline 1 & $\begin{array}{l}\text { NTOF1, BC-422, } 40 \times 20 \mathrm{~mm} \\
\text { with one side mirrored }\end{array}$ & $\sim 4.5 \mathrm{~m}$ & $\begin{array}{l}\text { Photek, } \\
\text { PMT240, } 10^{6}\end{array}$ & D2 & $10^{9}-10^{11}$ \\
\hline 2 & NTOF2, BC-422, $40 \times 20 \mathrm{~mm}$ & $\sim 4.5 \mathrm{~m}$ & $\begin{array}{l}\text { Photek, } \\
\text { PMT140, } 10^{3}\end{array}$ & D2 & $5 \times 10^{10}-5 \times 10^{12}$ \\
\hline 3 & NTOF3, BC-422Q, $40 \times 10 \mathrm{~mm}$ & $\sim 4.5 \mathrm{~m}$ & $\begin{array}{l}\text { Photek, } \\
\text { PMT140, } 100\end{array}$ & DT & $10^{11}-5 \times 10^{13}$ \\
\hline 4 & NTOF4, BC-422, $40 \times 10 \mathrm{~mm}$ & $\sim 4.5 \mathrm{~m}$ & PD040, 1 & DT & $10^{13}-10^{15}$ \\
\hline 5 & NTOF5, CVD 10 x 1 mm (E6) & $\sim 4.5 \mathrm{~m}$ & na & DT & $10^{13}-5 \times 10^{14}$ \\
\hline 6 & Multi-channel Scint. Detector & $\sim 20 \mathrm{~m}$ & Varies & Ignition & $10^{14}-2 \times 10^{19}$ \\
\hline 7 & CVD diamonds set & $\sim 20 \mathrm{~m}$ & na & Ignition & $10^{15}-2 \times 10^{19}$ \\
\hline
\end{tabular}

The NIF NTOF 1, 2, 3, \& 4 detectors are scintillator-PMT/PD assemblies and will have the same housing design, with only the scintillator and PMT/PD as variable parts [Figure 1]. Both scintillator and PMT are shielded from hard X-rays from all sides by $25 \mathrm{~mm}$ of lead. On the NIF we are expecting a tremendous X-ray signal and a harsh Electromagnetic Pulse (EMP) environment that will be increased significantly with the implementation of the Advanced Radiographic Capability (ARC) short pulse laser [4]. Because the X-rays arrive before the neutrons they may saturate the detector or simply dominate the signal to the point that a neutron pulse is undetectable. To eliminate this problem we apply a suppression gate to the PMT from the expected onset of the X-ray signal to just prior to the onset of the neutron signal. A PMT gating module will be placed inside or outside of the housing depending on results of ongoing EMP sensitivity tests.

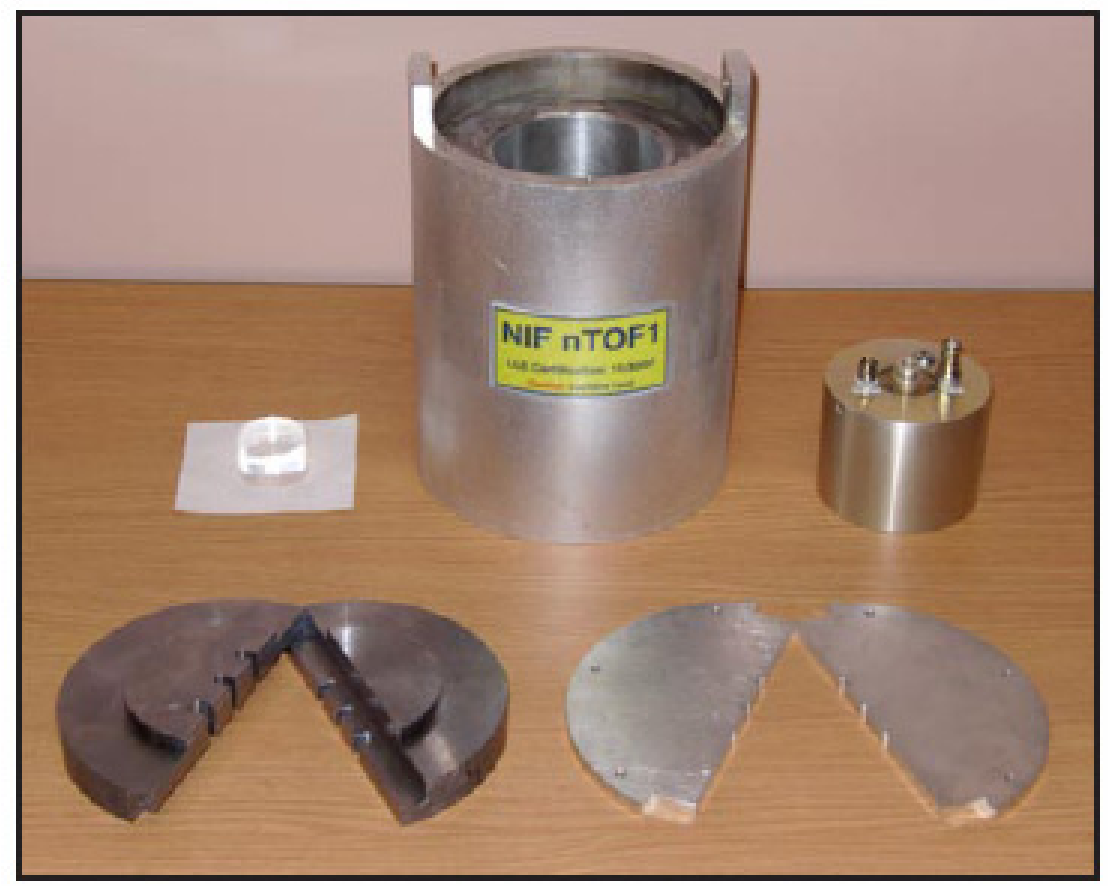

Figure 1. A disassembled NIF NTOF1 detector, showing the scintillator, PMT, shielding, and cap. 
The CVD diamond detectors are wafers of CVD diamond of varying diameter, thickness, and purity. Electrical contacts are laid on both sides of the wafer, and then the wafer is captured between two readout contacts that are part of the housing - one side to signal, the other to ground. CVD diamond detectors with different housing designs were tested and operated [5] on OMEGA for many years. Figure 2 shows a new housing design with high EMP mitigation that was developed for the NIF CVD diamond detectors. High voltage (HV) to these detectors is applied through a signal cable using a commercially available [6] bias T. After assembling the NIF CVD, diamond detectors were tested at $\mathrm{HV}$ two times larger than the operational $\mathrm{HV}$ value both at air pressure and in vacuum.

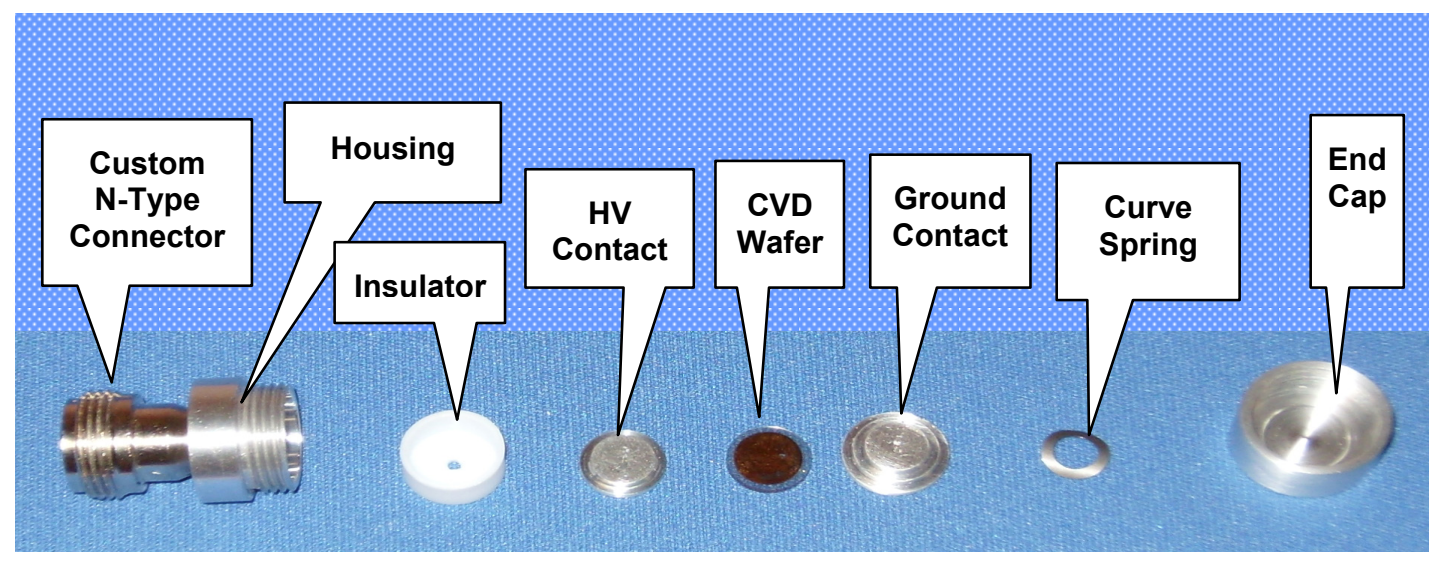

Figure 2. Physical assembly of NIF CVD diamond detector

While we tested the NIF NTOF detectors at several facilities, our conclusions are based on data from Titan and OMEGA. Titan is the most powerful and intense laser system of the Jupiter Laser Facility (JLF) [7] at LLNL. Capable of delivering a short laser pulse with petawatt intensity, Titan can provide an X-ray and EMP environment on the scale of the NIF with ARC, making it a good environment for testing detectors in harsh environment without neutrons present. On Titan, the NIF NTOF detectors were located $\sim 5.8 \mathrm{~m}$ from TCC. They were read out with $12 \mathrm{ft}$ of RG-142 co-axial cable running to $30 \mathrm{ft}$ of LMR 400 which was connected to a junction panel on an EMI shielded rack in another room behind $3 \mathrm{ft}$ of concrete. Inside the rack, LMR 400 cables were connected to $1 \mathrm{GHz}$ oscilloscopes, running at $5 \mathrm{GS} / \mathrm{s}$. We tested a variety of housing designs, with similar diamonds, under bias, at Titan. We found that the new NIF CVD diamond detector housing mitigated the EMP noise on Titan to below the digitization noise of the oscilloscope. At $5.8 \mathrm{~m}$ the X-ray yield is not detected by the CVD diamond wafers. However, the same X-ray signal produced a $\sim 50 \mathrm{~V}$ pulse in the NIF NTOF1 scintillator detector shown in Figure 3. To eliminate the X-ray signal we applied a $400 \mathrm{~ns}$ suppression gate to the PMT. This reduced the measured X-ray pulse on Titan to below the digitization noise of the oscilloscope [Figure 3]. This successful test demonstrated the possibility of using a gated PMT to eliminate the hard X-ray signal expected in the NIF NTOF detectors on NIF-ARC. The measured EMP signal from the NIF NTOF1 detector on Titan has an amplitude of $150 \mathrm{mV}$ at maximum, decaying to $20 \mathrm{mV}$ at the time of the neutron pulse. Although $20 \mathrm{mV}$ is acceptable level of the EMP noise for the NTOF1 detector, future studies and tests are planned in order to improve EMP mitigation of the NIF scintillator housing. 


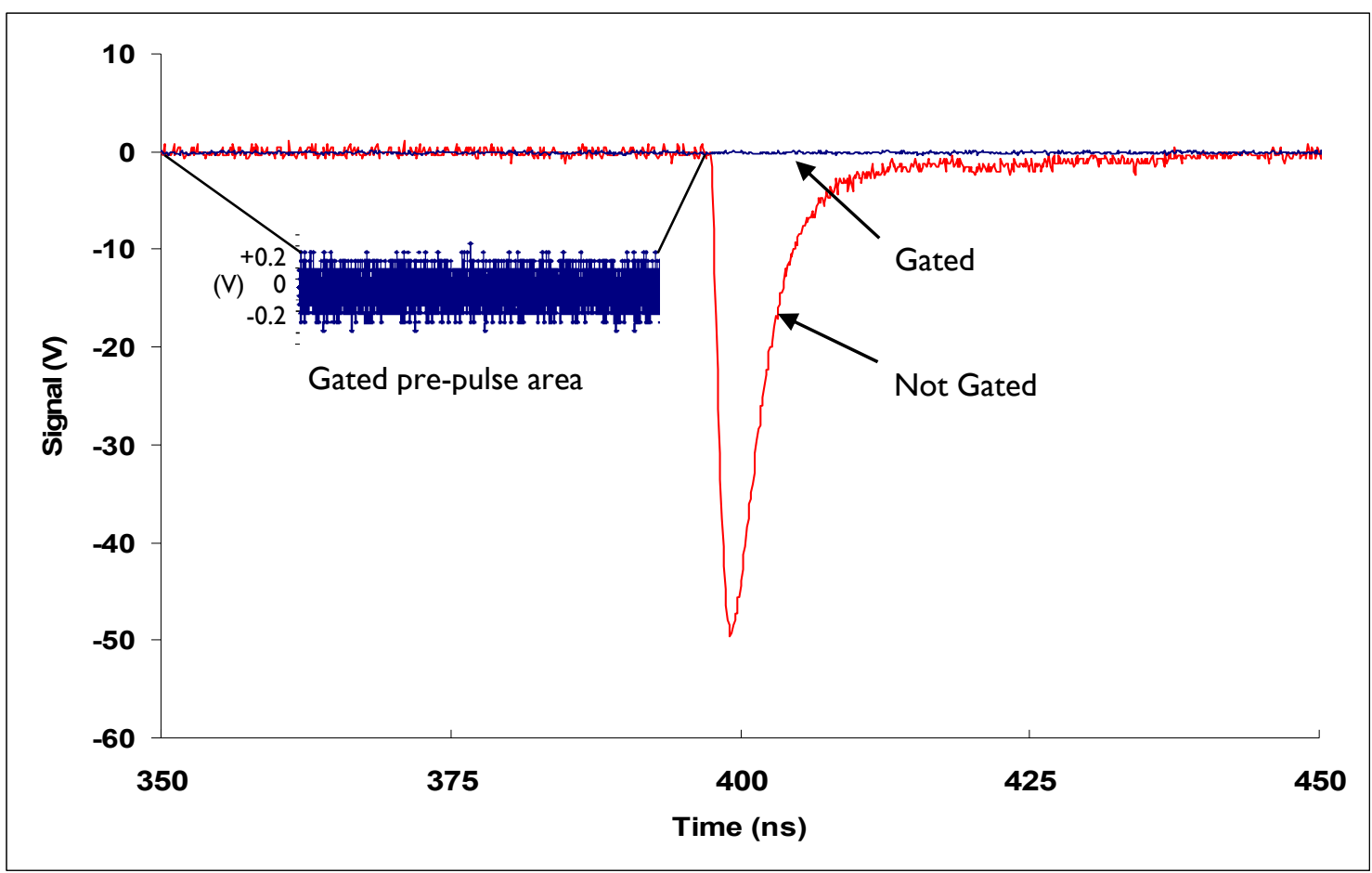

Figure 3. Hard X-ray signal measured by NIF NTOF1 with and without a gate on Titan.

\section{Neutron Calibration on OMEGA}

Two permanent locations have been established on OMEGA for calibrating the NIF NTOF detectors. One location at $5.16 \mathrm{~m}$ from TCC was designed for calibrations of the NIF scintillator detectors NTOF1 - NTOF4. The other location at $5.3 \mathrm{~m}$ from TCC is designed for calibration of the NIF CVD diamond detector NTOF5. The set of four CVD diamonds for the NIF NTOF7 detector will be calibrated one-by-one inside the OMEGA target chamber in a 10 inch manipulator. A procedure for the NTOF7 CVD diamond calibration was developed, tested, and is ready to use. The setup to calibrate the NIF 4-channel scintillator NTOF6 detector will be installed on OMEGA in 2008. Neutron calibrations of the NIF NTOF detectors were carried out on the OMEGA laser with the same cables, HV power supply, and digital oscilloscopes that were selected for operation on the NIF.

NIF NTOF $1 \& 3$ were calibrated over a number of OMEGA shot days to provide a range of neutron yield. Power was supplied by a Stanford Research Systems PS350 [8] high voltage power supply located in La Cave via a single high voltage cable. The signal was received from the detector by a $12 \mathrm{ft}$ RG142 cable to a $140 \mathrm{ft}$ LMR600 cable which was then connected to a Broadwave Technologies 4-Way signal splitter [9], and from there to a $1 \mathrm{GHz}, 5 \mathrm{GS} / \mathrm{s}$ oscilloscope. We find that the signal amplitude varies linearly with neutron yield [Figure 4]. 


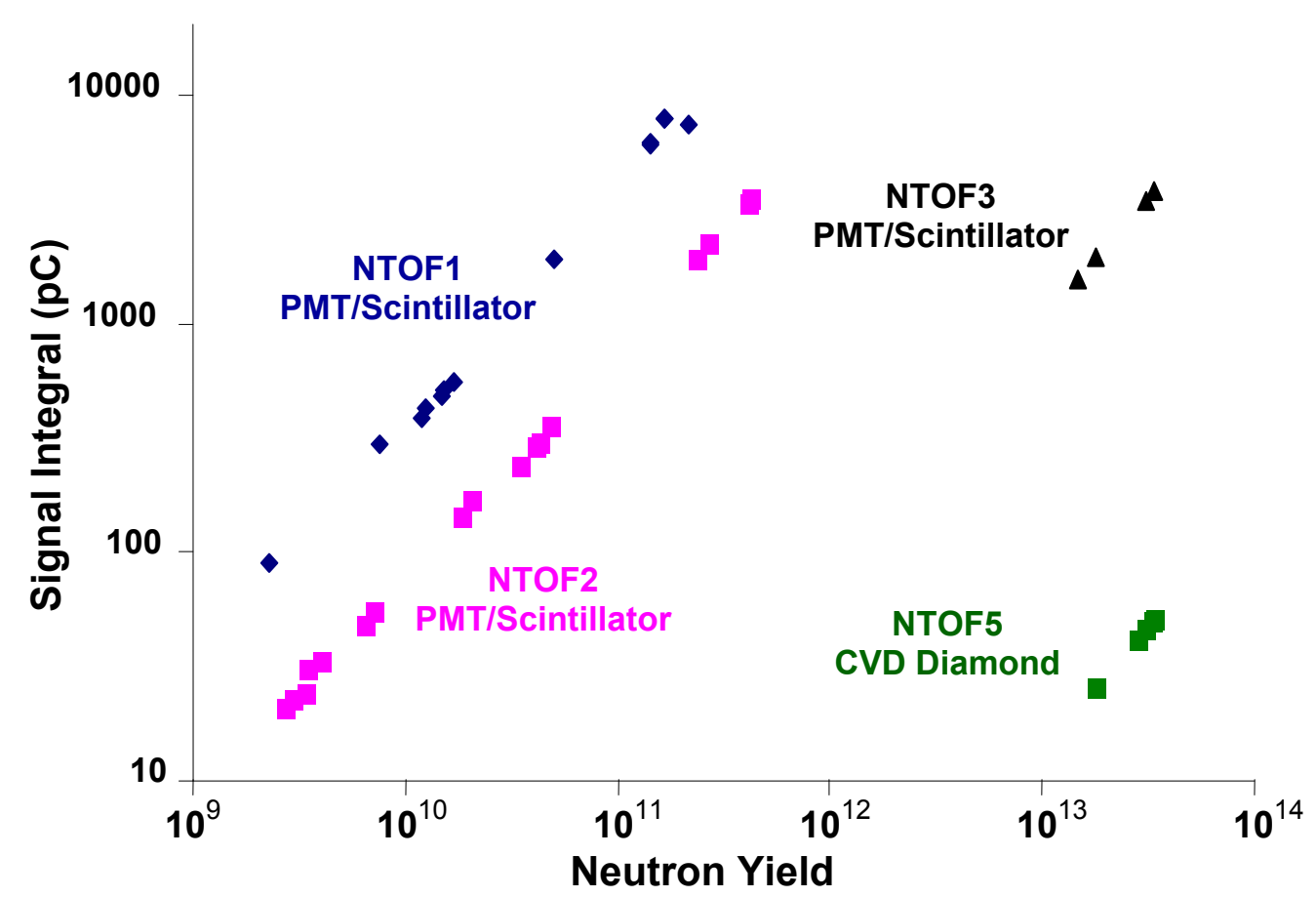

Figure 4. The NIF NTOF detectors show excellent linearity in neutron yield, and the varying sensitivity will allow use over the full required dynamic range.

NIF NTOF1 measured neutron yields from $10^{9}$ to $10^{11}$. The detector consists of a $40 \mathrm{~mm}$ diameter, $2.0 \mathrm{~mm}$ thick BC-422 [10] target-face mirrored scintillator coupled to a two-stage gated micro channel plate (MCP) Photek model PMT240 photomultiplier biased at $-3.8 \mathrm{kV}$. This results in a signal gain of $3 \times 10^{4}$. NIF NTOF3 measured neutron yields from $10^{11}$ to $5 \times 10^{13}$. The scintillator was a $40 \mathrm{~mm}$ diameter, $10 \mathrm{~mm}$ thick, (1\% benzophenone) quenched scintillator without any coating, coupled to a single stage MCP Photek model PMT140 photomultiplier biased at $-3.4 \mathrm{kV}$. This results in a signal gain of 100 . Tests were conducted with the PMT gate applied, and no effect on the detector system response was found.

The NTOF5 CVD diamond detector was calibrated with identical readout electronics, with the exception that the $140 \mathrm{ft}$ LMR600 signal cable also carried the high voltage to the detector via a Picosecond Pulse Labs Model $5531 \mathrm{HV}$ Bias Tee [6]. NIF NTOF5 was a $10 \mathrm{~mm}$ diameter, $1 \mathrm{~mm}$ thick, CVD diamond wafer encased in the NIF CVD housing design. During operation it was biased at $1.5 \mathrm{kV}$ and measured neutron yields up to $3 \times 10^{13}$, and has the range to measure up to $5 \times 10^{14}$. As shown in Figure 5, the pulse shapes are similar for the different detectors, with sensitivity being the relevant difference. 

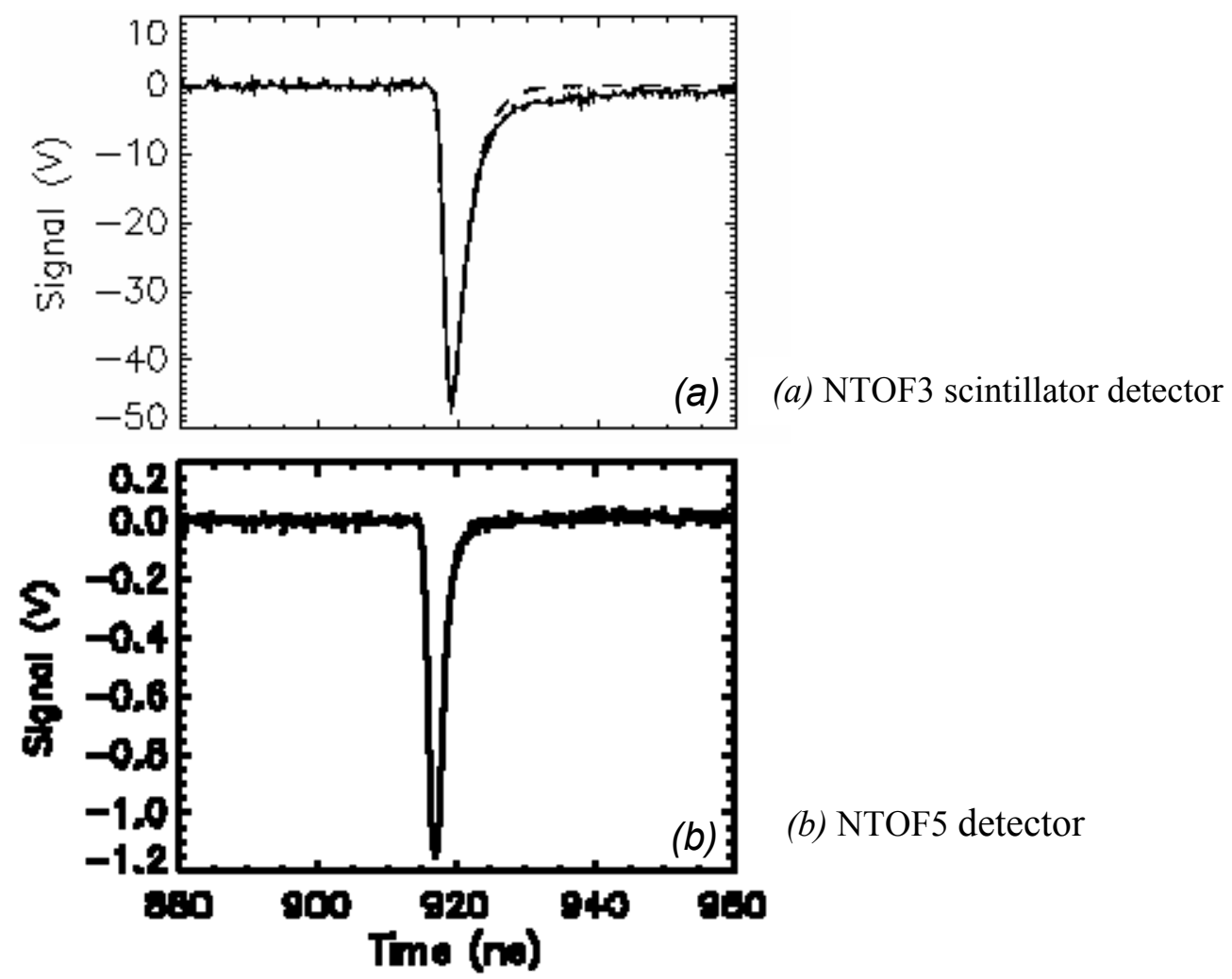

Figure 5. Neutron signal for OMEGA shot 50279 (DT) with yield $3.36 \times 10^{13}$ and $T_{i}=5.2 \mathrm{keV}$

The NIF NTOF detectors will also measure the implosion core ion temperature $\left(\mathrm{T}_{\mathrm{i}}\right)$ by first understanding the detector transfer function and then using a well known method of extracting the width of the neutron pulse [12]. We used this method to analyze the NTOF2 data, and in Figure 6 see that, as expected, the $\mathrm{T}_{\mathrm{i}}$ measured by the NIF NTOF detector corresponds to standard OMEGA measurements.

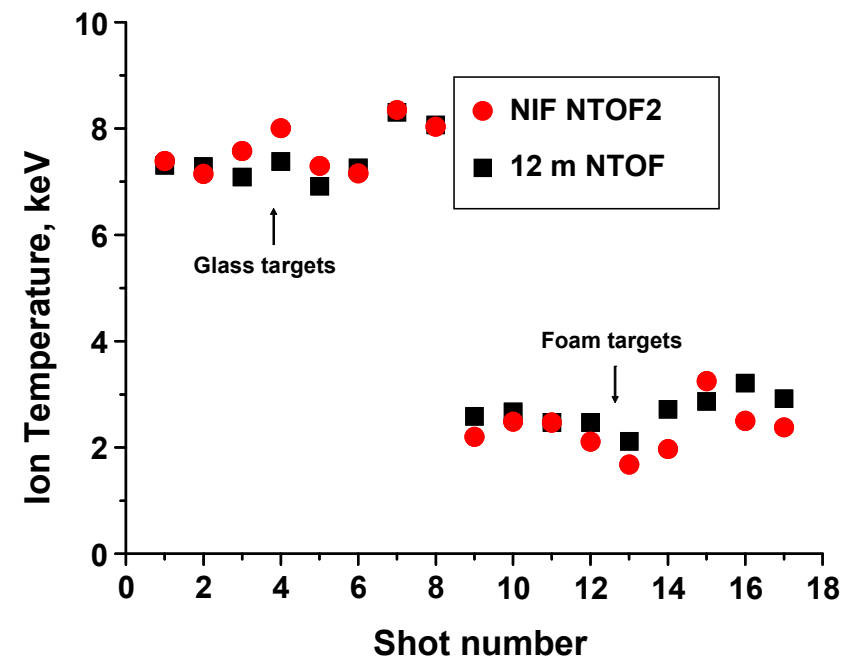

Figure 6: Core ion temperature measured by NIF NTOF2 corresponds well with $T_{i}$ measured by the standard $12 \mathrm{~m}$ NTOF detector at OMEGA. 


\section{Future Plans}

We have successfully tested and calibrated NIF NTOF detectors at several facilities and this work will continue at LLE. Having determined optimal housing design for the CVD detectors and the X-ray gating method for scintillator detectors, we will apply these methods to the NIF NTOF detectors to be located $20 \mathrm{~m}$ from TCC; these detectors will become the focus of development. We have already tested a scintillator in La Cave at LLE, $20 \mathrm{~m}$ from OMEGA TCC and have tested CVD diamond detectors in the OMEGA 10 inch diagnostic manipulators (TIMs), placing them $40 \mathrm{~cm}$ from OMEGA TCC. The neutron flux at this point will be similar to what we expect

to see on the NIF chamber wall. Furthermore, we will fully characterize the response functions of the NTOF detectors and examine the effect of the cable length on detector saturation. Plans are also in place for calibrating core ion temperature measurements made with these detectors. When the suite is complete, the current NIF NTOF dynamic range will have been extended from $10^{9}-10^{15}$ neutrons to $10^{9}-10^{19}$ neutrons.

\section{References}

[1] V. Yu. Glebov et al., Rev. Sci. Instrum. 75(10), 3558 (2004).

[2] E. I. Moses, Fusion Sci. Technol. 44, 11 (2003).

[3] V. Yu. Glebov et al., Rev. Sci. Instrum. 77, 10E715 (2006).

[4] E. I. Moses et al., J. Phys. IV France 133 (2006) 9-16.

[5] G. J. Schmid et al., Rev. Sci. Instrum. 74(3), 1828 (2003).

[6] Picosecond Pulse Labs, Inc. http://www.picosecond.com/objects/5531\%20SPEC-4040035.pdf

[7] J. A. Caird, J. D. Bonlie et al., Janus Intense Short Pulse (ISP) the Next Ultrahigh Intensity Laser at LLNL, Proceedings Intl. Conf. Ultrahigh Intensity Lasers 2004.

[8] Stanford Research Systems http://www.thinksrs.com/products/PS300.htm

[9] Broadwave Technologies http://www.broadwavetechnologies.com/ProductSpecSheets/PowerDividers/powerdividers.html

[10] Saint-Gobain Crystals http://www.detectors.saint-gobain.com/

[11] Photek, Ltd. http://www.photek.co.uk/products/detectors_photomultipliers.htm

[12] T. J Murphy et al., Rev. Sci. Instrum. 68(1), 610 (1997).

[13] T. R. Boehly et al., Opt. Commun. 133, 495 (1997).

This manuscript has been authored by National Security Technologies, LLC, under Contract No. DE-AC52-06NA25946 with the U.S. Department of Energy (DOE). The United States Government retains, and the publisher, by accepting the article for publication, acknowledges that the United States Government retains a nonexclusive, paid-up, irrevocable world-wide license to publish or reproduce the published form of this manuscript, or allow others to do so, for United States Government purposes. This manuscript was also authored by the University of Rochester with the New York State Energy Research and Development Authority supported by the U.S. DOE Office of Inertial Confinement Fusion under Cooperative Agreement No. DE-FC52-92SF19460. U.S. DOE support does not constitute endorsement of the views herein expressed. This work was done under the auspices of the U.S. DOE by Lawrence Livermore National Laboratory under Contract DE-AC52-07NA27344. 